Journal of Synchrotron Radiation

ISSN 0909-0495

\section{Speckle correlation as a monitor of X-ray free electron laser induced crystal lattice deformation}

\author{
Rajan Plumley, ${ }^{\text {a }}$ Yanwen Sun, ${ }^{\text {a Samuel Teitelbaum, }}$, b Sanghoon Song, ${ }^{a}$ \\ Takahiro Sato, ${ }^{a}$ Matthieu Chollet, ${ }^{a}$ Nan Wang, ${ }^{a}$ Aymeric Robert, ${ }^{a}$ Paul Fuoss, ${ }^{a}$ \\ Mark Sutton ${ }^{\mathrm{c}}$ and Diling Zhu ${ }^{\mathrm{a} *}$
}

\begin{abstract}
${ }^{a}$ Linac Coherent Light Source, SLAC National Accelerator Laboratory, 2575 Sand Hill Road, Menlo Park, CA 94025, USA, b PULSE Institute, SLAC National Accelerator Laboratory, 2575 Sand Hill Road, Menlo Park, CA 94025, USA, and 'Physics Department, McGill University, 845 Sherbrooke St W, Montréal, Quebec H3A 0G4, Canada. Correspondence e-mail: dlzhu@slac.stanford.edu
\end{abstract}

X-ray free electron lasers (X-FELs) present new opportunities to study ultrafast lattice dynamics in complex materials. While the unprecedented source brilliance enables high fidelity measurement of structural dynamics, it also raises experimental challenges related to the understanding and control of beam-induced irreversible structural changes in samples that can ultimately impact the interpretation of experimental results. This is also important for designing reliable high performance X-ray optical components. In this work, we investigate X-FEL beaminduced lattice alterations by measuring the shot-to-shot evolution of near-Bragg coherent scattering from a single crystalline germanium sample. We show that $\mathrm{X}$ ray photon correlation analysis of sequential speckle patterns measurements can be used to monitor the nature and extent of lattice rearrangements. Abrupt, irreversible changes are observed following intermittent high-fluence monochromatic $\mathrm{X}$-ray pulses, thus revealing the existence of a threshold response to X-FEL pulse intensity.
(C) 0000 International Union of Crystallography Printed in Singapore - all rights reserved

\section{Introduction}

From studies of condensed matter to biological systems, the exceptionally high brightness of X-FEL pulses has enabled obtaining information at unprecedented small and fast spatial and temporal regimes (Bostedt et al., 2016). However, that same quality also exacerbates the risk of incurring damages to the sample under investigation by the probing X-ray radiation itself. This technical challenge has been a recurring concern for $\mathrm{X}$ ray methods since their beginnings in the late nineteenth century (Slater, 1951; Henderson, 1995). FELs have for example demonstrated that intense X-ray pulses focused down to very small sizes can destroy materials in a single-shot and can even affect X-ray optical components (Hau-Riege et al., 2008; HauRiege et al., 2010; Koyama et al., 2013). This reinforces that it is essential to characterize and also understand FEL X-ray beam-induced damage process.

While the potential risk of incurring radiation damage is a concern for nearly all X-FEL experiments, its severity strongly varies on various parameters such as the modes of operation, the material of interest, and the X-ray measurement technique being employed. The seminal work by Neutze et al. described the key experimental concept of obtaining "diffraction-before-destruction" for the structure determination of macro biomolecules, in which the X-ray diffraction outruns the Coulomb explosion and ensuing disintegration of the molecule thanks to the femtosecond pulse duration and the 'instantaneous' nature of the X-ray scattering process (Neutze et al., 2000). This concept has also been extended to crystalline samples, and is further strengthened by the notion of "self-termination of diffraction" which addresses the faster than expected structural alteration by the extremely intense FEL pulses (Boutet et al., 2012; Barty et al., 2012). This also enables damage-free structural determination of biomolecules such as membrane proteins and metalloprotein that are otherwise too sensitive to cumulative damage effects induced by the effectively continuous radiation delivery of third-generation synchrotron sources (Kern et al., 2014).

In contrast to common conceptions, not all FEL experiments operate in the diffract-before-destroy regime. For example, ultrafast diffraction experiments probing excited structural dynamics in condensed matter typically use stroboscopic measurements where the sample receives repetitive localized exposures from X-ray pulses over hours (Trigo et al., 2013; Clark et al., 2013; Gerber et al., 2017). The instantaneous radiation dose must be limited to a low enough level to reduce the risk of cumulative irreversible modification to the sample that could change the dynamics under investigation. For X-ray photon correlation spectroscopy (XPCS) and in particular split-pulse XPCS, the probe-probe nature of the method imposes even more stringent requirement on the intensity of single pulse to minimize its impact on the dynamics being investigated (Lehmkühler et al., 2018; Möller et al., 2019). Carnis et al. have used X-FEL radiation to examine the relaxation dynamics of gold nanoparticles while also characterizing the 
eventual occurrence of sample damage. They demonstrated the feasibility of XPCS studies of soft matter materials at X-FEL beamlines with the caveat that experimental boundaries must be imposed to limit sample degradations (Carnis et al., 2014). To date, experimental efforts have mostly relied on empirical 'trial and error' approaches. Systematic understanding of sample damage mechanisms has been scarcely documented.

Another challenge unique to FEL sources is the intrinsic intensity fluctuation of SASE-generated X-ray pulses (Bonifacio et al., 1994). Not only does this further complicate the optimization of XPCS measurements, but if the beamline is operating in a configuration that experiences large shot-to-shot incident intensity variations, the sudden occurrence of a high intensity pulse could damage the sample or even beamline optics. For example, Koyama and coworkers at the SPring-8 angstrom compact free-electron laser facility (SACLA) have observed the single-shot ablation of thin films and substrates commonly used for X-ray optics applications by monochromatic hard $\mathrm{X}$-rays, observing a threshold response and imprint size directly correlated with to the X-ray pulse intensity (Koyama et al., 2013).

In fact, the so-called 'imprint' technique has been widely used as a way to quantify beam profiles of pulsed laser sources (Liu, 1982; Hau-Riege et al., 2010; Koyama et al., 2013). While useful, the method relied on the systematic examination of the dose-array exposed samples at a later time using high resolution optical or electron microscopy. With the commissioning of new X-FEL facilities with even higher brilliance and repetition rates underway, new techniques for monitoring the degradation of materials during the course of experiments will be crucial in identifying optimal measurement conditions. Hence, an on-the-fly, pulse-resolved method compatible with typical scattering experiment setups such that any occurrence of sample alteration can be event-correlated would be desired.

Over the past decades, XPCS studies have demonstrated that the two-time correlation function (TTCF) can be used to study subtle changes in the material structures and dynamics on small length scales with high sensitivity (Sanborn et al., 2011; Evenson et al., 2015). In this work we employ this method to investigate the creation and evolution of lattice disorder in a single-crystalline sample via TTCF analysis of sequential Xray speckle correlation measurements. We also show that by comparing FEL X-ray pulse intensities to their resultant lattice rearrangement effects (i.e., as manifested in the decorrelation of subsequent speckle patterns) can be used to determine the threshold for permanent lattice disorder rearrangement.

\section{Experimental setup and observations}

The experiment was performed at the X-ray Correlation Spectroscopy (XCS) instrument at the Linac Coherent Light Source (Alonso-Mori et al., 2015). As illustrated in Figure 1, an $\mathrm{X}$-ray beam with a photon energy of $9.5 \mathrm{keV}$ was monochromatized using a dual-channelcut four-bounce $\mathrm{Si}(220)$ monochromator (i.e., the fixed-delay branch of the compact split-delay system (Sun et al., 2019) was used) down to a bandwidth of $0.5 \mathrm{eV}$. The X-ray pulse duration was estimated to be approximately 50 fs. Pulse intensities were monitored in-situ by a photodiode measuring scattering from a Kapton foil between the two $\mathrm{Si}(220)$ channelcuts. Beryllium compound refractive lenses (CRLs) with a focal length of $0.9 \mathrm{~m}$ were used to focus the beam to approximately 2 micron at the sample location. The germanium single crystal was polished into a 10 degree wedge shape and mounted on a compact goniometer in air at room temperature. The wedge shape allows to adjust the sample thickness with a simple translation; thus enabling the adjustment of the balance between the signal level, speckle size, and speckle contrast. After locating the (111) Bragg peak, the sample was rotated by $1^{\circ}$ from the peak position around the vertical axis to avoid detector saturation. The detector samples a slice of the reciprocal space near the $\mathrm{Ge}(111)$ Brillouin zone center. Downstream of the sample, the scattered X-rays traveled through a vacuum flight path before being recorded by an ePix 100a detector at a distance of $5.5 \mathrm{~m}$ (Carini et al., 2016). The detector pixel size was $50 \times 50$ microns. Its position was centered near the peak of the initial thermal diffuse scattering near the Ge (111) Bragg reflection direction.

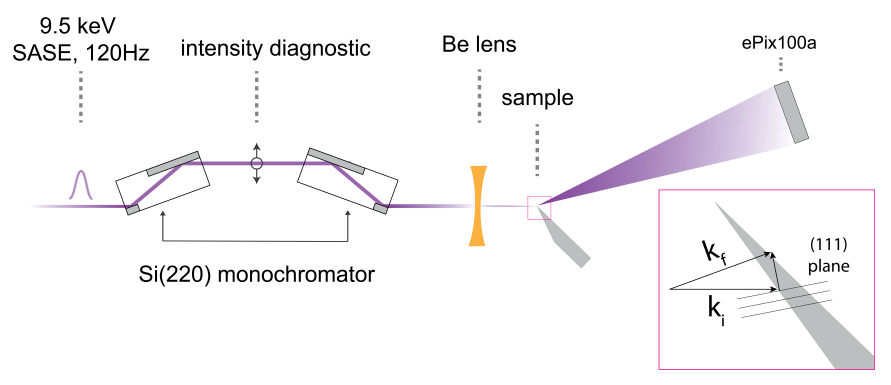

\section{Figure 1}

Schematics of the experimental setup. It consists of a 4-bounce $\operatorname{Si}(220)$ monochromator using a pair of channelcut crystals, slits for defining the beam trajectory, beryllium CRLs for focusing, and a diffractometer for orienting the sample. The scattering was collected with an ePix100a detector at a distance of 5.5 meter from the sample. The inset shows the geometry of the crystal 'wedge' and the orientation of the $\mathrm{Ge}(111)$ lattice plane, as well as the reciprocal space scattering geometry.

For perfect crystals at finite temperatures, near-Bragg intensities in the form of thermal diffuse scattering (TDS) are a manifestation of dynamic atomic displacements from the ideal crystal lattice positions (Warren, 1990). As larger amplitude static disorder is introduced into the crystal structure, additional diffuse scattering contributes to intensity increases near the zone center. When illuminated with coherent X-rays, these diffuse scattering contributions will appear as X-ray speckles (Sutton et al., 1991). The speckle pattern is a reflection of the arrangement of the disorder within the scattering volume. The measurements of the evolution of the speckle pattern thus carry information on how the disorder is changing from shot to shot. Figure 2 shows a typical sequence of near-Bragg coherent scattering patterns. Starting from a single crystal in its pristine condition, we observe a weak but relatively uniform distribution of TDS on average as shown in Figure 2(a). As the FEL irradiation continues, we observed step-wise increase in the total diffuse scattering intensity. We also observe clearly that the diffuse scattering 
form 'temporarily static' speckle patterns as shown in Figure 2 (b-d). The speckle patterns remained similar for typically a few tens of pulses and then rearranged significantly as seen in the difference between Figure 2 (c) and (d).
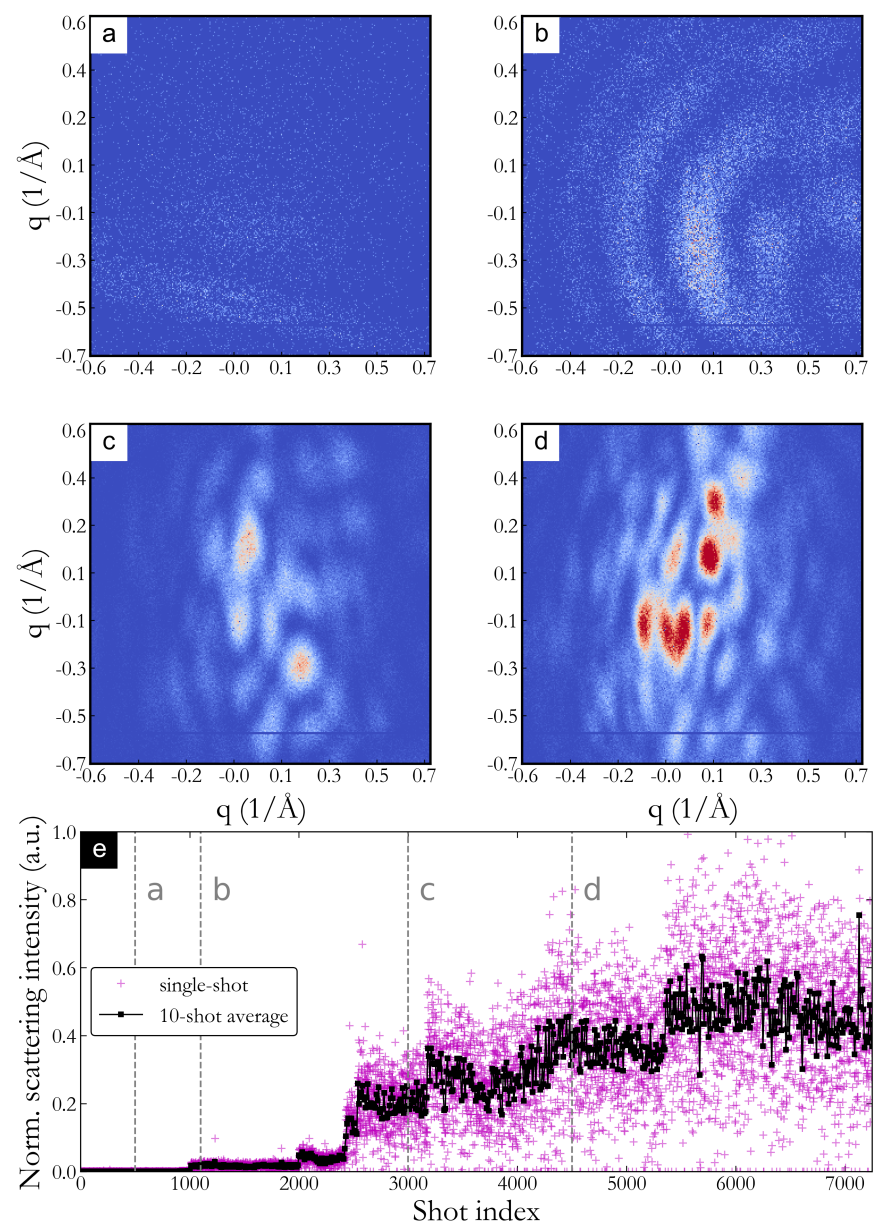

Figure 2

(a-d) Time-averaged near-Bragg scattering speckle patterns showing the evolution of speckles. (e) Single-shot (pink) and 10 shot integrated (black) speckle intensity as a function of total number of X-FEL pulse exposures, showing intensity increase in successive abrupt steps.

The step-wise sudden changes of the speckle pattern are triggered by so-called 'hot shots', as monochromatization of the SASE X-FEL beam presents high pulse intensity fluctuations (Zhu et al., 2014). On the other hand, we did not observe significant changes in speckle sizes nor a build up of DebyeScherrer ring, which suggests that the structural modification to the single crystal sample was still relatively subtle. This will be further discussed in a subsequent question.

To evaluate the sequence of scattering growth and speckle evolution, we employ the two-time correlation analysis method. The two-time correlation function is an extended formulation of the standard temporal correlation function $g^{(2)}(q, t)$ first used in dynamic light scattering experiments. The TTCF, which measures the statistical similarity between intensity measurements at any two points in time (Madsen et al., 2010), can be calculated as:

$$
g^{(2)}\left(q, t_{1}, t_{2}\right)=\frac{\left\langle I\left(q, t_{1}\right) I\left(q, t_{2}\right)\right\rangle_{\phi}}{\left\langle I\left(q, t_{1}\right)\right\rangle_{\phi}\left\langle I\left(q, t_{2}\right)\right\rangle_{\phi}},
$$

where $I(q, t)$ is the pixel intensity at the momentum transfer $q$ and time $t . \phi$ in the subscript of the bracket indicates averaging over the pixels where correlation shows negligible variation. It may be convenient to think about $g^{(2)}\left(q, t_{1}, t_{2}\right)$ as a moving evaluation of $g^{(2)}(q, t)$ such that $t_{0}$ is always along the $t_{1}=t_{2}$ diagonal. In these terms, the autocorrelation lag time is $t=t_{2}-t_{1}$. The time-boundary cases of the standard $g^{(2)}(q, t)$ extend to the two-time formulation as follows,

$$
g^{(2)}\left(q, t_{1}, t_{2}\right)= \begin{cases}1+\beta, & t_{1}=t_{2} \\ 1, & t_{2}-t_{1} \rightarrow \infty\end{cases}
$$

where $\beta$ is the optical contrast (Madsen et al., 2010). Note that the time unit in the context here are 'pulses' rather than the real time as we are interested in the beam induced pulse-to-pulse changes. From the coherent scattering patterns, we selected a $250 \times 250$ pixel region of interest (ROI) from the ePix100a detector images centered near the peak of the thermal diffuse scattering and covering a reduced momentum transfer range $q_{\phi} \rightarrow[-0.6,0.4] \AA^{-1}$. We define reduced momentum transfer with respect to TDS center of mass. While our TTCF ranges over 3200 sequential images, we only present the TTCF up to a lag time of $t=800$, as speckle decorrelations usually occur within the first few hundred shot increments and the total number of calculations to complete scales with the square of the number of images used.

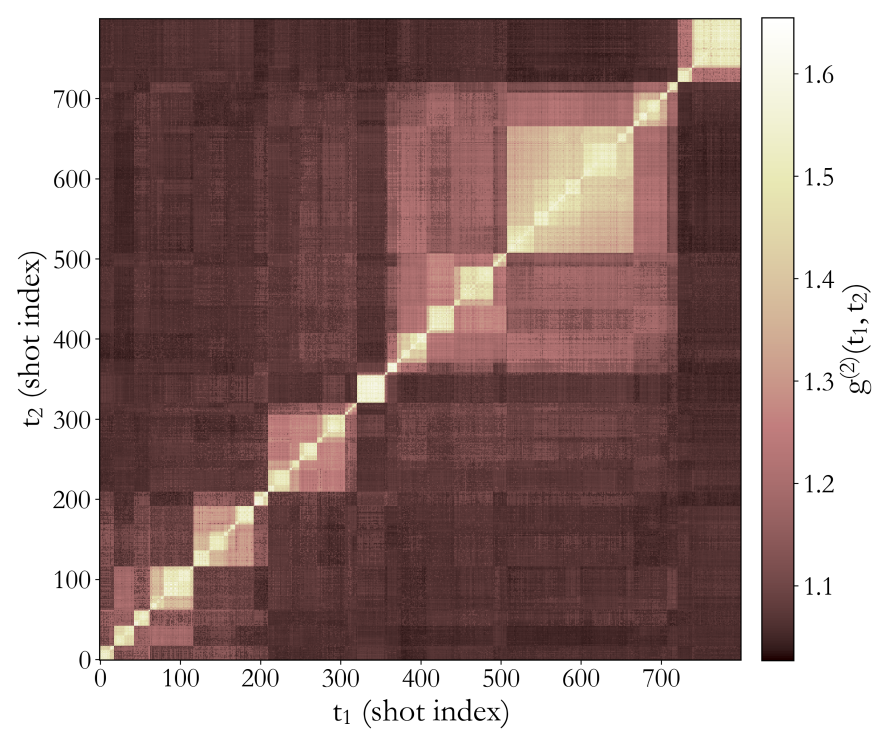

Figure 3

$800 \times 800$ crop of the two-time speckle correlation. Autocorrelation amplitudes lie along the $t_{1}=t_{2}$ diagonal and lag times can be scanned across vertical and horizontal directions.

Single-pulse exposures were used to calculate the TTCF so that any shot-to-shot evolution of the speckle pattern would not 
be time-averaged away. This required a high enough scattering intensity to enable sensitivity in speckle visibility measurement for each X-ray pulse (Falus et al., 2006). The data presented are collected from a slightly thicker part of the sample ( $\sim 20$ microns) with an X-ray pulse energy distribution that triggers relatively frequent speckle rearrangement during the experiment (every few seconds). We have also excluded scattering patterns from very low intensity pulses in our analysis as they were too weak to provide an accurate correlation measurement. Pulses at those intensities appeared to be below the threshold that would induce any observable speckle changes based on pulse-averaged measurements with attenuated beams. All speckle patterns were first thresholded at the detector pixel ADU value equivalent to half a photon in order to remove the readout noise of the dark region. Gaussian smoothing is then used to balance the intensity variation across the $q_{\phi}$ region of interest prior to the calculation of the correlation (Fluerasu et al., 2005). In total, 3200 sequential speckle pattern images from the ePix100a detector were used. Figure 3 shows an $800 \times 800$ section of the full two-time correlation function matrix.

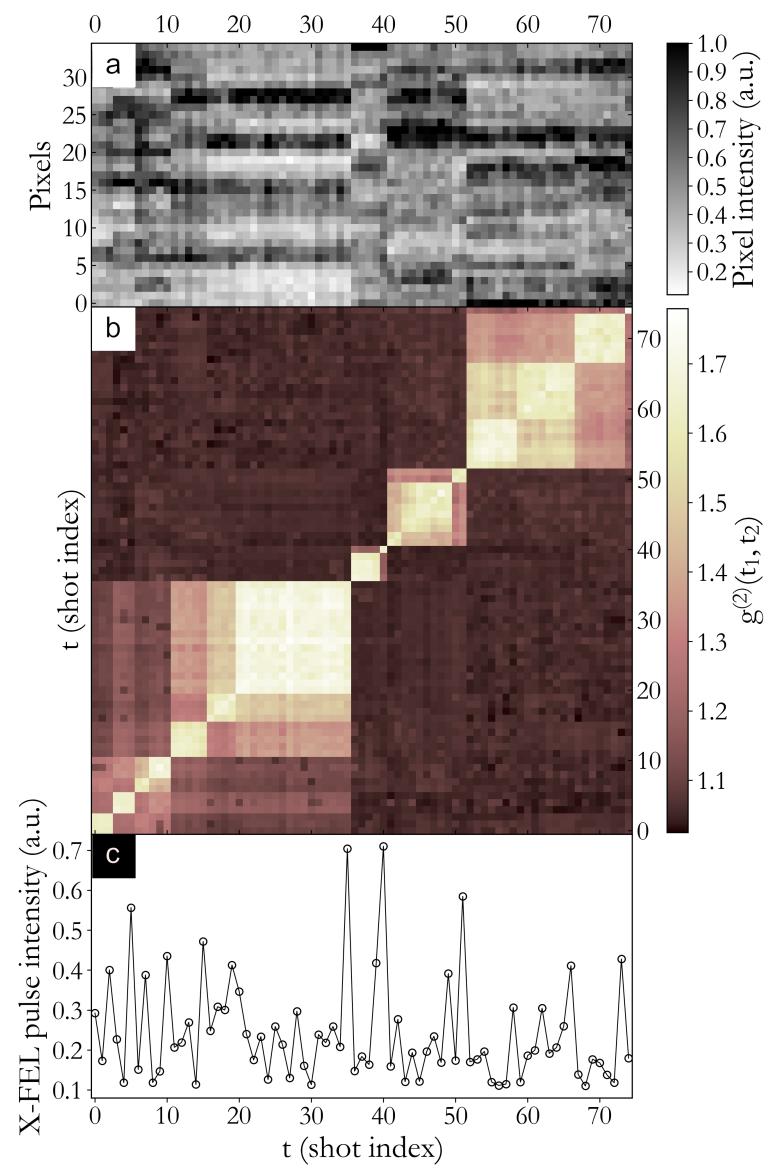

Figure 4

(a) A typical line-out of the speckle pattern as a function of number of shots. (b) a $75 \times 75$ ROI of the TTCF. (c) shot-by-shot X-ray pulse intensity measurements. One sees that the high intensity spikes aligns well with TTCF edges indicates conjunction between high-intensity laser pulses and abrupt speckle decorrelation on the next exposure event.
While two-time correlation analysis is predominantly used to study dynamics in non-equilibrium systems (Evenson et al., 2015; Lüttich et al., 2018; Madsen et al., 2010), our aim is to examine the abrupt changes of the speckle patterns resulting from stochastic variations in the X-FEL probe pulse intensity. Quick inspection of the TTCF in Figure 3 reveals a sequence of square tiles arranged along the autocorrelation diagonal. Tiles in the TTCF correspond to time-durations of minimal variation in the speckle intensity configuration, while their outward-facing edges characterize decorrelation within the time span between FEL exposure events. This signifies a rapid transformation of the atomic lattice structure similar to the two-time observations of martensitic transformations in cobalt by Sanborn and others (Sanborn et al., 2011). This behavior is examined more closely in Figure 4. Figure 4 (a) shows an example of speckle intensity line-out as a function of pulses, where abrupt transitions can be directly visualized. In the corresponding TTCF map shown in Figure 4 (b), we can see that the transitions of various degree maps very well to the touching corners of the square tiles of similar correlation values. All the abrupt transitions are also well matched to the shot-by-shot FEL pulse intensity measurement plotted on an aligned horizontal axis in Figure 4(c). Clearly, the extent of 'decorrelation' of the speckle pattern from the previous pattern is strongly related to the pulse energy of the previous pulse. To analyze this relationship more quantitatively, we define the speckle reconfiguration amplitude $\delta(t)$, that has the expression

$$
\delta(t)=g^{(2)}(t, t)-g^{(2)}(t, t-1)
$$

which is a measure of the change in $g^{(2)}$ amplitude at each time-step. Because a speckle pattern contains information that reflects the disordered structure producing it (Sutton et al., 1991), we can use $\delta(t)$ as a shot-by-shot monitor of the extent of lattice rearrangement that occurs in the irradiated portion of the sample. Equation ( 3 ) is evaluated along the $t=t_{1}=t_{2}$ diagonal for the full range of TTCF data. We use the event-index $t$ to gather pulse intensities $I_{0}(t-1)$ from the previous FEL shots. A plot of the speckle transition amplitude v.s. X-FEL pulse intensity is shown in Figure 5.

Figure 5 shows a threshold behavior of the speckle reconfiguration amplitude as a function of the X-FEL pulse intensity. While the energy deposition from the X-ray pulse is proportional to the measured pulse energy, there appears to be a minimum pulse energy required induce observable structural changes in the sample that led to the rearrangement of the speckle pattern. In the plot we show a least-squares linear fit to the data (with a fitting range for X-FEL pulse intensity larger than 0.30 a.u.) can be used to estimate the damaging threshold intensity of 0.27 a.u. However, while this in principle can be a rather sensitive probe to the structural damage threshold, we refrain from the discussion of the absolute threshold as it requires accurate measurement of the beam size, profile, as well as pulse energy which unfortunately was inadequate in this experiment. 


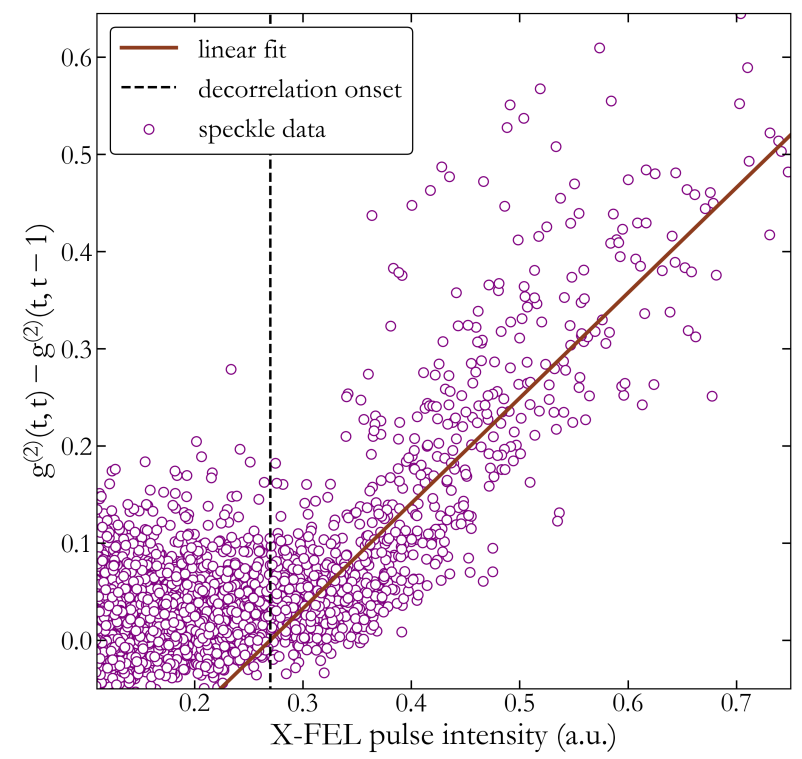

Figure 5

Speckle reconfiguration amplitudes $\delta$ evaluated over 3200 measurements v.s. $\mathrm{X}$-FEL pulse intensities of their previous shot index. A linear fit to determine the onset-intensity to drive speckle change is plotted (solid line), with the threshold indicated by the dotted line.

\section{Discussion \& Conclusion}

The threshold response of $\delta(t)$ favors the picture that the FEL beam induces a transient thermal gradient within the beam footprint and the center part of the irradiated sample reached a crystalline structural instability close to the melting point of the sample. The best estimate of the absolute pulse energy on the sample also put the threshold dose to within a factor of 2 of the melting dose of Ge at approximately $0.2 \mathrm{eV} /$ atom. The absence of the formation or even a precursor of Debye-Scherrer scattering ring indicates that the resolidification following the likelymelting of the sample did not result in an isotropic polycrystaline structure. It is also highly unlikely that the melted portion of the sample would enter an amorphous phase as that would lead to observation of reduced speckle sizes (as the effective volume of the sample contributing to the preferential scattering towards the $q$-region of interest will be larger). We thus hypothesize that the cooling rate must be sufficiently low such that a partially melted sample volume would have enough time to adiabatically recrystallize following the general lattice orientation of the surrounding cooler part of the crystal. This hypothesis is also supported by the lack of surface ablation features, damage craters, or other commonly-found damage features as carefully examined with both optical and scanning electron microscopes.

In conclusion, we have demonstrated a coherence-based, pulse-resolved technique for investigating irreversible FELinduced crystal structural alterations. Two-time correlation analysis of near-Bragg speckle measurements was shown to be a sensitive probe for monitoring the evolution of atomic structural disorder in a single crystal, which will be useful for further studies. We have also shown that crystalline structural damage thresholds relevant to specific reciprocal space regions can be determined by quantitative measurement of the decorrelation between successive speckle patterns and its correlation with the incident X-ray pulse intensities. This method is simple and can be made compatible with many hard X-ray scattering instruments at FEL facilities. With sufficient angular resolution on the detection side, it offers a much more sensitive monitor for the sample condition, as compared to the measurement of the average scattering.

Finally, the observation of intermittent rearrangement of the speckle patterns, and the correlation analysis protocol used to pin point the transitions, are relevant for data-reduction algorithms applicable to capturing rare events (Engels et al., 1999; Heinz \& Shpyrko, 2017), which is a new opportunity enabled by the upcoming MHz X-FEL sources such as LCLS-II and LCLSII-HE. We anticipate implementing TTCF-like analysis at the hardware level on the detector to down-select the transitional image arrays and thus play a key role in taking full advantage of high-brightness high-repetition X-FEL sources (Islambek et al., 2019).

\section{Acknowledgments}

RP acknowledges the computing support by Omar Qiujano. Use of the Linac Coherent Light Source (LCLS), SLAC National Accelerator Laboratory, is supported by the U.S. Department of Energy, Office of Science, Office of Basic Energy Sciences under Contract No. DE-AC02-76SF00515.

\section{References}

Alonso-Mori, R., Caronna, C., Chollet, M., Curtis, R., Damiani, D. S., Defever, J., Feng, Y., Flath, D. L., Glownia, J. M., Lee, S. et al. (2015). Journal of synchrotron radiation, 22(3), 508-513.

Barty, A., Caleman, C., Aquila, A., Timneanu, N., Lomb, L., White, T. A., Andreasson, J., Arnlund, D., Bajt, S., Barends, T. R. et al. (2012). Nature photonics, 6(1), 35.

Bonifacio, R., Salvo, L. D., Pierini, P., Piovella, N. \& Pellegrini, C. (1994). Nuclear Instruments and Methods in Physics Research Section A: Accelerators, Spectrometers, Detectors and Associated Equipment, 341(1), $181-185$.

URL: http://www.sciencedirect.com/science/article/pii/0168900294903441

Bostedt, C., Boutet, S., Fritz, D. M., Huang, Z., Lee, H. J., Lemke, H. T., Robert, A., Schlotter, W. F., Turner, J. J. \& Williams, G. J. (2016). Rev. Mod. Phys. 88, 015007.

URL: https://link.aps.org/doi/10.1103/RevModPhys.88.015007

Boutet, S., Lomb, L., Williams, G. J., Barends, T. R., Aquila, A., Doak, R. B., Weierstall, U., DePonte, D. P., Steinbrener, J., Shoeman, R. L. et al. (2012). Science, 337(6092), 362-364.

Carini, G., Alonso-Mori, R., Blaj, G., Caragiulo, P., Chollet, M., Damiani, D., Dragone, A., Feng, Y., Haller, G., Hart, P. et al. (2016). In AIP Conference Proceedings, vol. 1741, p. 040008. AIP Publishing.

Carnis, J., Cha, W., Wingert, J., Kang, J., Jiang, Z., Song, S., Sikorski, M., Robert, A., Gutt, C., Chen, S.-W. et al. (2014). Scientific reports, 4, 6017.

Clark, J., Beitra, L., Xiong, G., Higginbotham, A., Fritz, D., Lemke, H., Zhu, D., Chollet, M., Williams, G., Messerschmidt, M. et al. (2013). Science, 341(6141), 56-59.

Engels, M., Hoppe, B., Meuth, H. \& Peters, R. (1999). In ISCAS'99. Proceedings of the 1999 IEEE International Symposium on Circuits and Systems VLSI (Cat. No. 99CH36349), vol. 5, pp. 160163. IEEE.

Evenson, Z., Ruta, B., Hechler, S., Stolpe, M., Pineda, E., Gallino, I. \& Busch, R. (2015). Phys. Rev. Lett. 115, 175701.

URL: https://link.aps.org/doi/10.1103/PhysRevLett.115.175701

Falus, P., Lurio, L. \& Mochrie, S. (2006). Journal of synchrotron radiation, 13(3), 253-259. 
Fluerasu, A., Sutton, M. \& Dufresne, E. M. (2005). Physical review letters, 94(5), 055501.

Gerber, S., Yang, S.-L., Zhu, D., Soifer, H., Sobota, J. A., Rebec, S., Lee, J. J., Jia, T., Moritz, B., Jia, C., Gauthier, A., Li, Y., Leuenberger, D., Zhang, Y., Chaix, L., Li, W., Jang, H., Lee, J.-S., Yi, M., Dakovski, G. L., Song, S., Glownia, J. M., Nelson, S., Kim, K. W., Chuang, Y.-D., Hussain, Z., Moore, R. G., Devereaux, T. P., Lee, W.-S., Kirchmann, P. S. \& Shen, Z.-X. (2017). Science, 357(6346), 71-75.

URL: https://science.sciencemag.org/content/357/6346/71

Hau-Riege, S. P., London, R. A., Bionta, R. M., Soufli, R., Ryutov, D., Shirk, M., Baker, S. L., Smith, P. M. \& Nataraj, P. (2008). Applied Physics Letters, 93(20), 201105.

URL: https://doi.org/10.1063/1.3021081

Hau-Riege, S. P., London, R. A., Graf, A., Baker, S. L., Soufli, R., Sobierajski, R., Burian, T., Chalupsky, J., Juha, L., Gaudin, J., Krzywinski, J., Moeller, S., Messerschmidt, M., Bozek, J. \& Bostedt, C. (2010). Opt. Express, 18(23), 23933-23938.

URL: http://www.opticsexpress.org/abstract.cfm?URI=oe-1823-23933

Heinz, T. \& Shpyrko, O., (2017). Opportunities for basic energy research at the frontiers of xfel ultrafast science.

Henderson, R. (1995). Quarterly Reviews of Biophysics, 28(2), 171193.

Islambek, A., Yang, K., Li, W. \& Li, K. (2019). arXiv preprint arXiv: 1906.06042.

Kern, J., Hattne, J., Tran, R., Alonso-Mori, R., Laksmono, H., Gul, S., Sierra, R. G., Rehanek, J., Erko, A., Mitzner, R., Wernet, P., Bergmann, U., Sauter, N. K., Yachandra, V. \& Yano, J. (2014). Philosophical Transactions of the Royal Society B: Biological Sciences, 369(1647), 20130590.

URL: https://royalsocietypublishing.org/doi/abs/10.1098/rstb.2013.05

Koyama, T., Yumoto, H., Senba, Y., Tono, K., Sato, T., Togashi, T., Inubushi, Y., Kim, J., Kimura, T., Matsuyama, S. et al. (2013). In Journal of Physics: Conference Series, vol. 463, p. 012043. IOP Publishing.
Lehmkühler, F., Valerio, J., Sheyfer, D., Roseker, W., Schroer, M. A., Fischer, B., Tono, K., Yabashi, M., Ishikawa, T. \& Grübel, G. (2018). IUCrJ, 5(6), 801-807.

URL: https://doi.org/10.1107/S2052252518013696

Liu, J. M. (1982). Opt. Lett. 7(5), 196-198.

URL: http://ol.osa.org/abstract.cfm?URI=ol-7-5-196

Lüttich, M., Giordano, V. M., Le Floch, S., Pineda, E., Zontone, F., Luo, Y., Samwer, K. \& Ruta, B. (2018). Physical review letters, 120(13), 135504.

Madsen, A., Leheny, R. L., Guo, H., Sprung, M. \& Czakkel, O. (2010). New Journal of Physics, 12(5), 055001.

Möller, J., Sprung, M., Madsen, A. \& Gutt, C. (2019). IUCrJ, 6(5), 794-803.

Neutze, R., Wouts, Remco, v. d. S. D., Weckert, E. \& Hajdu, J. (2000). Nature, 406, 752-757.

Sanborn, C., Ludwig, K. F., Rogers, M. C. \& Sutton, M. (2011). Physical review letters, 107(1), 015702.

Slater, J. C. (1951). Journal of Applied Physics, 22(3), 237-256. URL: https://doi.org/10.1063/1.1699937

Sun, Y., Wang, N., Song, S., Sun, P., Chollet, M., Sato, T., van Driel, T. B., Nelson, S., Plumley, R., Montana-Lopez, J., Teitelbaum, S. W., Haber, J., Hastings, J. B., Baron, A. Q. R., Sutton, M., Fuoss, P. H., Robert, A. \& Zhu, D. (2019). Opt. Lett. 44(10), 2582-2585.

URL: http://ol.osa.org/abstract.cfm?URI=ol-44-10-2582

Sutton, M., Mochrie, S., Greytak, T., Nagler, S., Berman, L., Held, G. \& Stephenson, G. (1991). Nature, 352(6336), 608.

Trigo, M., Fuchs, M., Chen, J., Jiang, M. P., Cammarata, M., Fahy, S., Fritz, D. M., Gaffney, K., Ghimire, S., Higginbotham, A. \& others (2013). Nature Physics, 9, 790-794.

Warren, B. E. (1990). X-ray Diffraction. Courier Corporation.

Zhu, D., Feng, Y., Stoupin, S., Terentyev, S. A., Lemke, H. T., Fritz, D. M., Chollet, M., Glownia, J., Alonso-Mori, R., Sikorski, M. et al. (2014). Review of Scientific Instruments, 85(6), 063106. 